\title{
Performance and Energy Analysis of OpenMP Runtime Systems with Dense Linear Algebra Algorithms
}

\author{
João V. F. Lima*, Issam Raïs ${ }^{\S}$, Laurent Lefèvre ${ }^{\S}$, Thierry Gautier ${ }^{\S}$ \\ *UFSM, Brazil \\ $\S$ Univ. Lyon, Inria, CNRS, ENS de Lyon, Univ. Claude-Bernard Lyon 1, LIP, France
}

\begin{abstract}
In this paper, we analyse performance and energy consumption of four OpenMP runtime systems over a NUMA platform. We present an experimental study to characterize OpenMP runtime systems on the three main kernels in dense linear algebra algorithms (Cholesky, LU and QR) in terms of performance and energy consumption. Our experimental results suggest that OpenMP runtime systems can be considered as a new energy leverage. For instance, a $\mathrm{LU}$ factorization with concurrent write extension from libKOMP achieved up to 1.75 of performance gain and $\mathbf{1 . 5 6}$ of energy decrease.
\end{abstract}

\section{Introduction}

Energy-efficiency is one of the four major challenges that should be overcome in the path to exascale computing [1]. Despite improvements in energy-efficiency, the total energy consumed by supercomputers is still increasing due to the even quicker increase in computational power. High energy consumption is not only a problem of electricity costs, but it also impacts greenhouse emissions and dissipating the produced heat can be difficult. As the ability to track power consumption becomes more commonplace, with some job schedulers supporting tracking energy use [2], soon users of HPC systems may have to consider both how many CPU hours they need and how much energy.

Energy budget limitation imposes a high pressure to the HPC community making energy consideration a prominent research field. Most of the gain will come from technology by providing more energy efficient hardware, memory and interconnect. Nevertheless, recent processors integrate more and more leverages to reduce energy consumption (e.g. classical DVFS, deep sleep states) and low level runtime algorithms provide orthogonal leverages (e.g. dynamic concurrency throttling). However few of these leverages are integrated and employed in today local level software stack such as middleware, operating system or runtime library. Due to the complexity of this statement, we restricted our investigation to local node energy consumption by HPC OpenMP applications.

OpenMP is an API standard to express parallel portable programs. Most of controls are implementation defined and rely on the specific OpenMP programming environment used. The OpenMP standard does not impose any constraint on implementations. Even if there are more precise specifications, e.g. mapping of threads to cores, it is very tricky to precisely control performance or energy consumption using what OpenMP specification proposes [3]. Previous works have dealt with a specific OpenMP runtime [4], [5], [6], [7], [8], [9] that may be difficult to generalize to other OpenMP runtime systems without strong development effort. To the knowledge of the authors, there is no related work comparing OpenMP runtime systems in order to analyse performance and energy consumption.

In this paper, we analysed performance and energy consumption of four OpenMP runtime systems over a NUMA system. We restrict our experiments on three dense linear algebra algorithms: Cholesky, LU and QR matrix factorizations. Source codes are based on KASTORS [10] benchmark suite and the state of the art PLASMA library using its new OpenMP implementations [11] that rely on OpenMP tasks with data dependencies.

The contributions of this paper are:

- We present early experiments of performance and energy consumption over the dependent tasks model proposed by OpenMP.

- We report early comparisons of OpenMP runtime systems in order to present the respective gains with respect to one of the criteria.

- We observed that a LU factorization with concurrent-write access mode achieved up to 1.75 in performance gain and 1.56 in energy over original LU algorithm.

The remainder of the paper is organized as follows. Section 2 presents the related work. Section 3 gives some details of the OpenMP task programming model and an overview about five runtime implementations. Our experimental results are presented in Section 5. Finally, Section 6 and Section 7, respectively, present the discussion and conclude the paper.

\section{Related Work}

Other works use the simplicity proposed by OpenMP to vary the number of threads, for energy efficiency. Authors in [12] and [13] defend the Dynamic Concurrency Throttling (DCT) and underline the fact that using OpenMP to control the number of threads could be energy efficient, depending on the algorithm or the chosen hardware. 
Previous works show that various energy behaviors of computing nodes are possible through various leverages (DVFS, DCT, etc). But none of the previous work focus on OpenMP runtime systems as a leverage. None of the previous work dealt with the energy-performance trade-off and thus underlined possible variability concerning energy and performance for existing runtime systems. Thus, to the knowledge of the authors, no related work were trying to compare several OpenMP runtime libraries together for various representative workloads, as presented in our paper.

We use state of the art PLASMA library [11], on three main kernels in dense linear algebra (Cholesky, LU and QR factorizations), that implements dependent tasks model. This model is new and never addressed in related works. In [4], [5] the authors based their experiments using the BOTS [14] benchmarks that require only the independent tasks.

\section{OpenMP Task programming model and im- plementations}

In 2013 the OpenMP Architecture Review Board introduced in the OpenMP revision 4.0 a new way of expressing task parallelism using OpenMP, through the task dependencies. This section introduces the task dependency programming model targeted by the selected benchmark suites. We also present how the model is implemented in various runtime libraries.

\subsection{Dependent task model}

OpenMP dependent task model allows to define dependencies between tasks using declaration of accesses to memory with in,out, or inout. Two tasks are independent (or concurrent) if and only if they do not violated the data dependencies of a reference sequential execution order ${ }^{1}$.

Figure 1 illustrates a LU factorization based on PLASMA [11]. The programmer declares tasks and the accesses in, inout they made to a memory region (here only lvalue or memory reference, i.e. pointer).

The OpenMP library computes tasks and dependencies at runtime, and schedules concurrent tasks on the available processors. The strategy for task dependencies and task scheduling depends on the runtime implementation. Nevertheless, their implementations impact the performance and the energy consumption. Moreover, the absence of precise OpenMP specification about the task scheduling algorithm is the key point to allow research to improve performance and energy efficiency with implementation concerns.

\subsection{Runtime system implementations}

Table 1 summarizes the properties of four OpenMP runtime systems.

libGOMP is the OpenMP runtime that comes with the GCC compiler. Dependencies between tasks are computed

1. OpenMP does not allows variable renaming to suppress output and anti-dependencies.

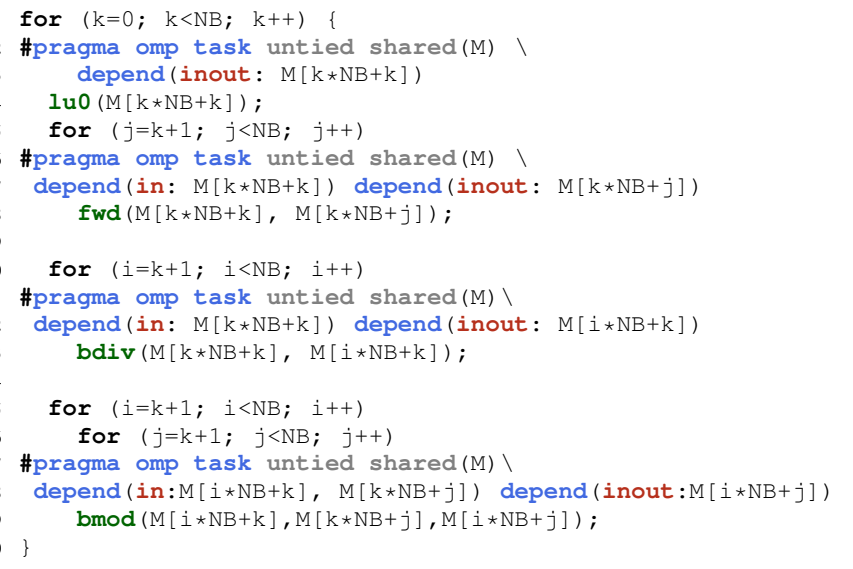

Figure 1. LU factorization with OpenMP dependent task.

TABLE 1. CHARACTERISTICS OF OPENMP RUNTIME SYSTEMS

\begin{tabular}{cccc}
\hline Name & Dependencies & Task Scheduling & Remarks \\
\hline libGOMP & hash table & centralized list & task throttling \\
\hline libOMP & hash table & work stealing & bounded dequeue \\
\hline XKaapi & hash table* & $\begin{array}{l}\text { non blocking } \\
\text { work stealing }\end{array}$ & task affinity \\
\hline libKOMP & $\begin{array}{c}\text { resizable } \\
\text { hash table }\end{array}$ & $\begin{array}{l}\text { non blocking } \\
\text { work stealing }\end{array}$ & $\begin{array}{c}\text { task affinity } \\
\text { concurrent write }\end{array}$ \\
\hline
\end{tabular}

through a hash table that map data (pointer) to the last task writing the data. Ready tasks are pushed into several scheduling dequeues. The main dequeue stores all the tasks generated by the threads of a parallel region. Tasks seem to be inserted after the position of their parent tasks in order to keep an order close to the sequential execution order. Because threads share the main dequeue, serialization of operations is guaranteed by a pthread mutex which is a bottleneck for scalability. To avoid overhead in task creation, libGOMP implements a task throttling algorithm that serialize task creation when the number of pending tasks is greater than a threshold proportional to the number of threads.

libOMP was initially the proprietary OpenMP runtime of Intel for its $\mathrm{C}, \mathrm{C}++$ and Fortran compilers. Now it is also the target runtime for the LLVM/Clang compiler and sources are open to community. libOMP manages dependencies in the same way that libGOMP by using a hash table. Memory allocation during task creation relies on a fast thread memory allocator. libOMP task scheduling is based on Cilk almost non blocking work stealing algorithm [15], but dequeue operations are serialized using locks. Nevertheless, it implies distributed deques management with high throughput of dequeue operations. libOMP also implements a task throttling algorithm by using bounded size dequeue.

XKaapi [16] is a task library for multi-CPU and multiGPU architectures which provides binary compatible library with libGOMP [17]. Task scheduling is based on the almost non blocking work stealing algorithm from Cilk [15] with extension to combine steal requests in order to reduce overhead in stealing [18]. Moreover, XKaapi computes de- 
pendencies on steal request, which is a perfect application of the work first principle to report overhead in task creation to critical path. The XKaapi based OpenMP runtime also has support to some OpenMP extensions such as task affinity [19] that allows to schedule tasks on NUMA architecture, and to increase performance by reducing memory transfer and thus memory energy consumption.

libKOMP [20] is a redesign of [17] on a top of the Intel runtime libOMP. It includes following features coming mainly from XKaapi: the dequeue management and work stealing with request combining; task affinity specific work stealing heuristic; a dynamically resized hash map that avoid high conflicts when finding dependencies for large tasks' graph; and tracing tool based on the OpenMP OMPT API; and finally a task concurrent write extension with a Clang modification ${ }^{2}$ to provide the OpenMP directive clause. This latter extension allows better parallelism and was used in one of our LU benchmark and it very closed of the task reduction feature currently under discussion in the OpenMP architecture review board.

\subsection{Discussion}

In our study of the mentioned OpenMP runtime systems, none of them include energy leverage such as thread throttling or DVFS. Nevertheless, their different task scheduling algorithms may impact energy efficiency. The main dequeue accesses in libGOMP serialize threads using a POSIX mutex. On Linux the mutex will block waiting threads after short period of active polling which ensure that few core cycles will be waste in the synchronisation.

On the other hand, libOMP, XKaapi and libKOMP work stealing actively poll dequeues until the program ends or a task is found. In order to reduce activity during polling, libOMP and libKOMP may block threads after an unsuccessful search of work by $200 \mathrm{~ms}$ (default value). Once work is found, all threads are waked up.

\section{Tools and Methods}

This section details the hardware configurations we experimented on and the OpenMP runtime systems we compared. We also give hints about the methodology used to process the collected data using statistical tools R.

\subsection{Evaluation platform}

Our experimental platform was the Brunch machine composed of four NUMA nodes with one Intel Xeon E78890 processor each (total 4 processors) and 24 cores per processor ( 96 cores total) running at $2.2 \mathrm{GHz}$, and $1.5 \mathrm{~TB}$ of main memory. The operating system on Brunch is a Debian with Linux kernel 4.9 .13 with 3 over 5 C-State activated (idle states: POLL C1-BDW C1E-BDW) with turbo-boost on and performance governor.

2. http://gitlab.inria.fr/openmp/clang
TABLE 2. BLOCK SIZE FOR EACH ALGORITHM AND MATRIX SIZE.

\begin{tabular}{rccc}
\hline & \multicolumn{3}{c}{ BRUNCH } \\
Matrix size & Cholesky & LU & QR \\
\cline { 2 - 4 } 8192 & 224 & 160 & 224 \\
16384 & 288 & 224 & 480 \\
32768 & 352 & 352 & 352 \\
\hline
\end{tabular}

\subsection{Software description}

4.2.1. Benchmarks. We used kernels from two benchmark suites: the KASTORS [10] benchmark suite and an OpenMP-parallelized PLASMA version [11]. Both benchmark suites tackle the same computational problems but use different algorithms in some cases. KASTORS was built from PLASMA 2.6.0 (released in dec. 2013) at a time when PLASMA parallelism was supported by a specific task management library called QUARK.

We focused our study on three dense linear algebra kernels:

- A Cholesky factorization (dpotrf);

- A LU factorization (dgetrf);

- A QR factorization (dgegrf).

Cholesky factorization algorithms in both the benchmark suites are the same. All these linear algebra kernels we used rely on the BLAS routines, we used the implementation of OpenBLAS version 0.2.19. Table 2 shows the block size configuration on each execution test for the three machine platforms.

4.2.2. Runtime Systems. We compared the following runtime systems during our experiments:

- LibGOMP - the OpenMP implementation from GNU that comes with GCC 6.3.0.

- LibOMP - a port of the Intel OpenMP open-source runtime to LLVM release 4.0.

- LibKOMP [20] - a research runtime system, based on the Intel OpenMP runtime, developed at INRIA. It offers several non-standard extensions to OpenMP. We evaluate the concurrent write $(\mathrm{CW})$ feature in our experiments coupled with Cilk T.H.E work stealing protocol. We make experiments with version efb $6 \mathrm{c} 36^{3}$.

- XKAAPI [16] - research runtime system developed at INRIA. It has lightweight task creation overhead, and it offers several non-standard extensions to OpenMP [17] We evaluate its version ef a $5 \mathrm{fdf}^{4}$.

\subsection{Energy measurement methodology}

Since several metrics have to be considered depending on the objective, we consider performance (GFlop/s) and energy consumption (energy-to-solution). GFlop/s is measured by the each benchmark itself: it corresponds to the

\footnotetext{
3. Git hash from https://gitlab.inria.fr/openmp/libkomp

4. From http://kaapi.gforge.inria.fr
} 
algorithmic count of the number of floating point operations over the elapsed time, using fact that matrix-matrix product does not rely on a fast algorithm such as Strassen like algorithm. Times are get using the Linux clock_gettime function with CLOCK_REALTIME clock.

We employed two sources of data acquisition for energy measurement. One was the Intel RAPL (Running Average Power Limit) feature that exposes the energy consumption of several components on the chip (such as the processor package and the DRAM) through MSRs (Model Specific Registers). Due to access limitation of MSRs on the tested system, we designed a small tool querying periodically the RAPL counters based on LIKWID [21]: Energy consumption for the whole package (PWR_PKG_ENERGY), for the cores (PWR_PP 0_ENERGY), for the DRAM (PWR_DRAM_ENERGY), as well as the core temperature (TEMP_CORE). The tool gets the counter values periodically and associate them with a timestamp.

The Brunch machine has been instrumented through a high-accuracy (error $<0.1 \%$ ) power meter LMG450 from Zimmer $^{5}$. The power meter is attached to the wall outlet and it measures the entire energy consumption of the machine, including power supply, disk, motherboard, etc. The output of the power meter (energy and power) periodically send data recorded with a timestamp. The Brunch machine measured $176 \mathrm{~W}$ to the wall outlet on the same period of inactivity. The idle power is a mean of 20 minutes of inactivity on the system with a process monitoring the RAPL counters.

\subsection{Experimental methodology}

All benchmarks are composed of two steps: the first allocates and initializes a matrix; the second step is the computation. We report execution time only from the computation step. Each experiment is repeated at least 30 times, each computation on a newly random matrix (as implemented by the benchmark). All the processes are spawned within the context of numactl to distribute memory pages among the NUMA nodes. In parallel of the computation, we monitor the system by collecting various energy counters from RAPL and the watt meter plugged on the wall outlet.

For each computation we collect the performance (GFlop/s) timestamped by the beginning and the end of the computation. This two timestamps are used in data postprocessing to compute energy consumed by the computation between the two timestamps. Values are interpolated by linear function if missing in the collected energy values sampled periodically. Post-processing employs R script to compute energy per computation and to output basic statistic for each configuration. In our experimental results, energy values are the mean computed among the at least $30 \mathrm{com}-$ putations of each configuration.

5. https://www.zes.com/en/Products/Precision-Power-Analyzer/ LMG450

\section{Experimental results}

The presented runtime systems have been experimented on the two benchmark suites presented in section 4.2.1. We build two configurations of libKOMP using two sets of options [20]. On the following libkomp refers to libKOMP configured with T.H.E Cilk work stealing queue and requests combining protocol; and libkomp_cw is the same configuration than libkomp with addition to support concurrent write extension used in the KASTORS LU code dgetrf [10].

\subsection{Runtime impact}

Figure 2 shows performance and energy results with three matrix sizes and over all machine resources available. We used as reference the GCC runtime to compute the difference over the other three runtime systems, represented on the bar plots by a percentage value.

These results suggest that libkomp and xkaapi attained the best performance results in most cases. Xkaapi outperformed others with Cholesky and QR on smaller input sizes (8192 and 16384), while libkomp had better results with input size 32768 on both algorithms. The LU algorithm with $\mathrm{CW}$ showed significant improvement compared to other runtime systems (up to $107.4 \%$ over gcc), followed by state of the art LU with libkomp.

In energy our experiments suggest that gcc had generally better energy efficiency on the three benchmarks, except for LU with $\mathrm{CW}$. Besides, if we compare only the original runtime systems coming with gcc and Intel compilers, it seems that gcc configuration performed better in performance and energy. This can be explained by the passive list scheduling in gcc, which is less reactive than work-stealing based strategies. Regarding LU energy results, the CW LU version with libkomp_cw reduced energy up to $24 \%$ (RAPL) and $31 \%$ (ZES) over gcc. Other runtime systems had lower energy efficiency than gcc.

\subsection{Focus on $\mathbf{L U}$ factorization}

Thanks to the concurrent write, the LU algorithm with libkomp_cw runtime had more parallelism than other runtime systems due to the $\mathrm{CW}$ algorithm extension based on KASTORS [10]. Figure 3 illustrates a Gantt execution from the LU factorization using libkomp_cw.

On the LU factorization, even if $\mathrm{CW}$ generates more parallelism, the algorithm has poor efficiency and threads are frequently idle. The Gantt diagram on all the 96 cores of brunch illustrates long periods of inactivity. Gcc is the only runtime where threads lock common dequeue to get task. Linux will put these lightweight process idle which is captured by energy sensors (ZES on brunch and by using RAPL counters). If we do not consider libkomp_cw's algorithmic variant, then gcc is the best runtime in term of energy consumption for LU factorization. This is not true for runtime systems based on task scheduling by work stealing such as libKOMP, libOMP or XKaapi which are very active process that consume energy. 

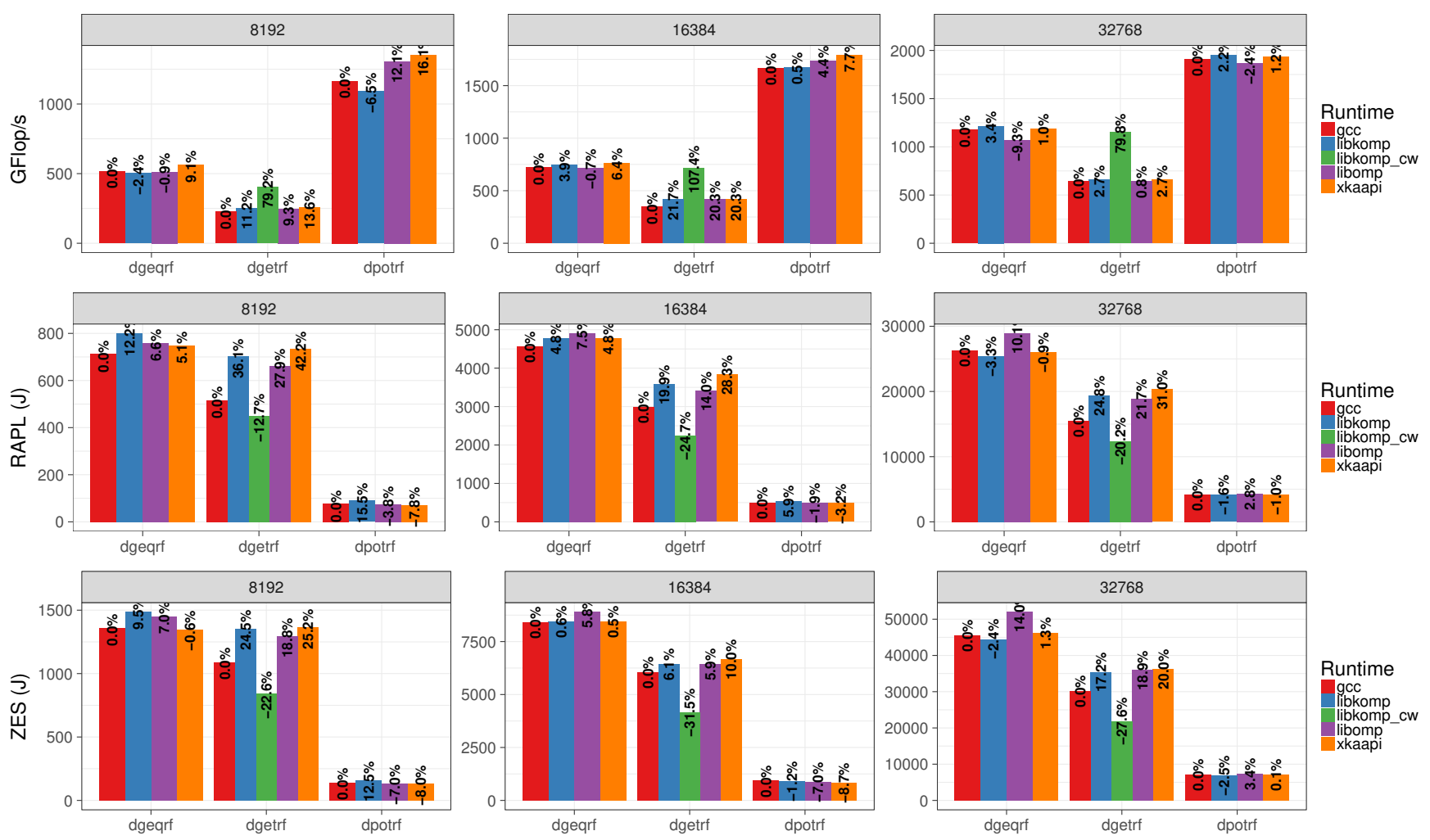

Figure 2. Performance (GFLop/s) and energy (Joules) results of Cholesky, LU and QR over the Brunch machine. All percentages on top of bar plots are the difference of current runtime over GCC runtime.

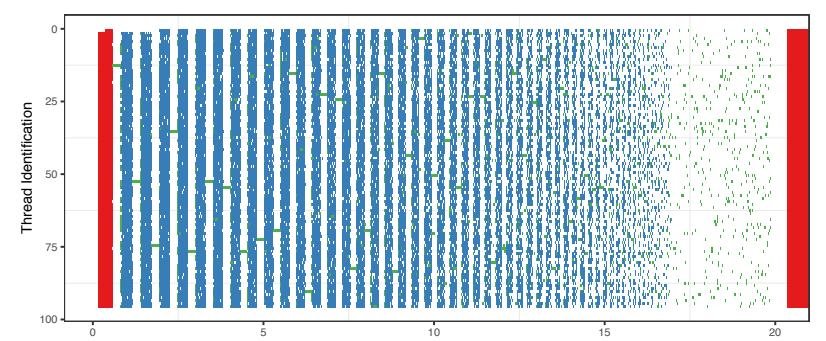

Figure 3. Gantt of KASTORS LU algorithm from libKOMP CW.

\section{Discussion}

Majority of best configurations from Figure 2 were runtime systems using work-stealing based scheduling. On fine grain problems, xkaapi and libkomp were generally better. These results can be explained by the smaller task creation overhead on xkaapi than libkomp and gcc.

The difference between libomp and libkomp is the new features we add into the original Intel libomp runtime: the lightweight work stealing algorithm from Cilk and the request combining protocol from xkaapi. These features not only impact performance but also the way tasks are scheduled: it suppresses the bounded dequeue limitation that may degenerate task creation into task serialization. It means that at runtime a thread may be forced to execute immediately tasks for which no or less affinity exist. Without bounded size dequeue, a thread that completes a task will always activate one of the successors following a data flow relationship producer-consumer, thus sharing a data resident into cache; or the thread becomes idle and try to steal tasks. We will investigate by more finer experiments the exact impact of these additions in libkomp.

On LU factorization where algorithmic variant libkomp_cw was the best, it was followed by xkaapi and libkomp on performance. LU factorization is a relevant code with inactivity sections from the dependencies imposed by the algorithm, mainly due to a search of pivot and swap of elements. This optimized algorithm allowed to increase performance while energy is decreased due to libkomp_cw runtime and concurrent write OpenMP extension [10]. Nevertheless, the platform characteristic, and especially its memory network, had also an impact on both performance and energy consumption.

Without these algorithm variants, LU factorization code consumes less energy using gcc runtime. In gcc the synchronization between threads on the shared task dequeue resource wastes less cycles. A work stealing based runtime may have interest to incorporate part of [22] in which is used to lower the speed of threads that are not in the critical path with a warranty on performance. One of the big challenges is the design of adaptive OpenMP runtime capable to saving energy on short delays of inactivity. 


\section{Conclusion}

In this paper, experiments with four production based OpenMP runtime systems on the three main kernel in dense linear algebra were conducted on a NUMA platform. We showed that OpenMP runtime is a new leverage for controlling energy. Our experimental results suggest that small algorithmic and runtime improvements may allow performance gains up to 1.75 and thus reducing the energy by a factor of 1.56. Besides, GCC runtime was energy efficient in some cases due to synchronizations over a shared task dequeue.

Future works include an extension of our experimental comparison over a wide range of architectures, including Intel KNL many-core. In addition, we will evaluate the impact on performance and energy of different configurations of leverages to control processor consumption and activity as available at operating system level.

\section{Acknowledgments}

This work is integrated and supported by the ELCI project, a French FSN ("Fond pour la Société Numérique") project that associates academic and industrial partners to design and provide a software environment for very high performance computing.

\section{References}

[1] K. Bergman, S. Borkar, D. Campbell, W. Carlson, W. Dally, M. Denneau, P. Franzon, W. Harrod, K. Hill, J. Hiller et al., "Exascale computing study: Technology challenges in achieving exascale systems," 2008.

[2] X. Yang, Z. Zhou, S. Wallace, Z. Lan, W. Tang, S. Coghlan, and M. E. Papka, "Integrating dynamic pricing of electricity into energy aware scheduling for hpc systems," in Proceedings of the International Conference on High Performance Computing, Networking, Storage and Analysis, ser. SC '13. New York, NY, USA: ACM, 2013, pp. 60:1-60:11.

[3] M. A. S. Bari, N. Chaimov, A. M. Malik, K. A. Huck, B. Chapman, A. D. Malony, and O. Sarood, "Arcs: Adaptive runtime configuration selection for power-constrained openmp applications," in 2016 IEEE International Conference on Cluster Computing (CLUSTER), Sept 2016, pp. 461-470.

[4] A. K. Porterfield, S. L. Olivier, S. Bhalachandra, and J. F. Prins, "Power measurement and concurrency throttling for energy reduction in openmp programs," in 2013 IEEE International Symposium on Parallel Distributed Processing, Workshops and Phd Forum, May 2013, pp. 884-891.

[5] A. Nandamuri, A. M. Malik, A. Qawasmeh, and B. M. Chapman, "Power and energy footprint of openmp programs using openmp runtime api," in Proceedings of the 2Nd International Workshop on Energy Efficient Supercomputing, ser. E2SC '14. Piscataway, NJ, USA: IEEE Press, 2014, pp. 79-88.

[6] C. Su, D. Li, D. S. Nikolopoulos, K. W. Cameron, B. R. d. Supinski, and E. A. León, "Model-based, memory-centric performance and power optimization on numa multiprocessors," in 2012 IEEE International Symposium on Workload Characterization (IISWC), Nov 2012, pp. 164-173.
[7] A. Marathe, P. E. Bailey, D. K. Lowenthal, B. Rountree, M. Schulz, and B. R. de Supinski, High Performance Computing: 30th International Conference, ISC High Performance 2015, Frankfurt, Germany, July 12-16, 2015, Proceedings. Cham: Springer International Publishing, 2015, ch. A Run-Time System for Power-Constrained HPC Applications, pp. 394-408.

[8] C. Lively, X. Wu, V. Taylor, S. Moore, H.-C. Chang, and K. Cameron, "Energy and performance characteristics of different parallel implementations of scientific applications on multicore systems," Int. J. High Perform. Comput. Appl., vol. 25, no. 3, pp. 342-350, Aug. 2011.

[9] D. Li, B. R. de Supinski, M. Schulz, K. Cameron, and D. S Nikolopoulos, "Hybrid mpi/openmp power-aware computing," in 2010 IEEE International Symposium on Parallel Distributed Processing (IPDPS), April 2010, pp. 1-12.

[10] P. Virouleau, P. Brunet, F. Broquedis, N. Furmento, S. Thibault, O. Aumage, and T. Gautier, "Evaluation of OpenMP Dependent Tasks with the KASTORS Benchmark Suite," in 10th International Workshop on OpenMP, ser. IWOMP'14. Springer, 2014, pp. 16 29.

[11] A. YarKhan, J. Kurzak, P. Luszczek, and J. Dongarra, "Porting the plasma numerical library to the openmp standard," International Journal of Parallel Programming, pp. 1-22, 2016.

[12] M. Curtis-Maury, J. Dzierwa, C. D. Antonopoulos, and D. S Nikolopoulos, "Online strategies for high-performance power-aware thread execution on emerging multiprocessors," in Proc. 20th IEEE International Parallel Distributed Processing Symposium, April 2006.

[13] A. K. Porterfield, S. L. Olivier, S. Bhalachandra, and J. F. Prins, "Power measurement and concurrency throttling for energy reduction in openmp programs," in Parallel and Distributed Processing Symposium Workshops \& PhD Forum (IPDPSW), 2013 IEEE 27th International. IEEE, 2013, pp. 884-891.

[14] A. Duran, X. Teruel, R. Ferrer, X. Martorell, and E. Ayguade, "Barcelona openmp tasks suite: A set of benchmarks targeting the exploitation of task parallelism in openmp," in Proc. of the 2009 International Conference on Parallel Processing, ser. ICPP '09. Washington, DC, USA: IEEE Computer Society, 2009, pp. 124-131.

[15] M. Frigo, C. E. Leiserson, and K. H. Randall, "The implementation of the cilk-5 multithreaded language," SIGPLAN Not., vol. 33, no. 5, pp. 212-223, May 1998.

[16] T. Gautier, J. V. F. Lima, N. Maillard, and B. Raffin, "Xkaapi: A runtime system for data-flow task programming on heterogeneous architectures," in Proceedings of the 2013 IEEE 27th International Symposium on Parallel and Distributed Processing, ser. IPDPS '13. Washington, DC, USA: IEEE Computer Society, 2013, pp. 1299 1308 .

[17] F. Broquedis, T. Gautier, and V. Danjean, "Libkomp, an efficient openmp runtime system for both fork-join and data flow paradigms," in Proceedings of the 8th International Conference on OpenMP in a Heterogeneous World, ser. IWOMP'12. Berlin, Heidelberg: SpringerVerlag, 2012, pp. 102-115.

[18] M. Tchiboukdjian, N. Gast, and D. Trystram, "Decentralized list scheduling," Annals of Operations Research, vol. 207, no. 1, pp. 237$259,2013$.

[19] P. Virouleau, F. Broquedis, T. Gautier, and F. Rastello, "Using data dependencies to improve task-based scheduling strategies on numa architectures," in Proceedings of the 22Nd International Conference on Euro-Par 2016: Parallel Processing - Volume 9833. New York, NY, USA: Springer-Verlag New York, Inc., 2016, pp. 531-544.

[20] T. Gautier and P. Virouleau, "New libkomp library. http://gitlab.inria.fr/openmp/libkomp," Jan. 2015.

[21] J. Treibig, G. Hager, and G. Wellein, "LIKWID: lightweight performance tools," CoRR, vol. abs/1104.4874, 2011.

[22] H. Ribic and Y. D. Liu, "Energy-efficient work-stealing language runtimes," SIGARCH Comput. Archit. News, vol. 42, no. 1, pp. 513528, Feb. 2014. 